\title{
Endocervical Adenocarcinoma In Situ Phenotype with Ovarian Metastasis
}

\author{
Hyun-Soo Kim · Yeon Seung Chung · Moon Sik Kim · Hyang Joo Ryu · Ji Hee Lee \\ Department of Pathology, Severance Hospital, Yonsei University College of Medicine, Seoul, Korea
}

Adenocarcinoma in situ (AIS) of the endocervix is a precursor of usual type endocervical adenocarcinoma. By definition, endocervical AIS normally involves simple columnar epithelium only and does not invade the cervical stroma. ${ }^{1}$ Endocervical AIS exhibits a variable anatomic distribution, extending along the endocervical canal for several centimeters, and sometimes involving the entire circumference of the cervix. ${ }^{1}$ Extension of endocervical AIS proximal to the internal orifice is uncommon and metastatic involvement of unilateral or bilateral ovaries by endocervical adenocarcinoma is even rarer. However, several studies have reported a subset of endocervical tumors with minimal to no evidence of stromal invasion manifesting as metastatic ovarian tumors. ${ }^{2,3}$ Distinguishing this metastatic disease from a primary ovarian tumor is difficult because ovarian lesions are typically large, unilateral, confined to the ovary, and well differentiated with mucinous or endometrioid-like histology, which are features consistent with a primary ovarian tumor. ${ }^{2}$ The metastatic nature of these lesions can be confirmed by comparing the human papillomavirus (HPV) type to that of the endocervical tumors. ${ }^{4}$ In this report, we demonstrate a case of endocervical AIS with no evidence of stromal invasion involving the unilateral ovary and simulating a primary ovarian tumor.

\section{CASE REPORT}

A 45-year-old woman presented with a left ovarian mass 65

\section{Corresponding Author}

Hyun-Soo Kim, MD, PhD

Department of Pathology, Severance Hospital, Yonsei University College of Medicine,

50-1 Yonsei-ro, Seodaemun-gu, Seoul 03722, Korea

Tel: +82-2-2228-1794, Fax: +82-2-362-0860, E-mail: hyunsookim@yuhs.ac

Received: September 14, 2018 Revised: December 6, 2018

Accepted: December 16, 2018 months following diagnosis of endocervical AIS (Fig. 1A) from a punch biopsy and treated with an extended abdominal hysterectomy. She underwent left salpingo-oophorectomy and the left ovarian mass measured $7.0 \mathrm{~cm}$ in diameter, had a smooth surface, and was solid and cystic. The inner surface of the cystic lesion varied from smooth to rough with several nodules. Histologically, the tumor displayed confluent glandular architectures with primarily endometrioid-like (Fig. 1B) and focally mucinous (Fig. 1C) differentiation. Nuclei were enlarged and hyperchromatic with occasional mitotic figures and apoptotic bodies (Fig. 1D). There was no stromal invasion. The ovarian tumor was thought to represent an independent borderline endometrioid tumor. We reviewed slides obtained from the hysterectomy specimen, and concerns were raised for metastasis due to the histological similarity between the endocervical and ovarian lesions. Immunostaining for p16 and HPV genotyping were performed. Both endocervical (Fig. 1E) and ovarian (Fig. 1F) lesions exhibited strong and diffuse p16 expression and had identical HPV type 45. Based on these findings, the left ovarian tumor was diagnosed as metastatic endocervical adenocarcinoma. This study was approved by the Institutional Review Board of Severance Hospital with a waiver of informed consent (4-2018-0724).

\section{DISCUSSION}

When histologically similar malignant tumors occur at two locations, it is difficult to determine whether each is an independent primary tumor, or whether one represents a metastasis from the other. ${ }^{5}$ The concurrence of endocervical adenocarcinoma and ovarian tumor is quite uncommon, and the diagnostic difficulties encountered have received little attention. The majority of cases with ovarian involvement occur in advanced-stage endo- 

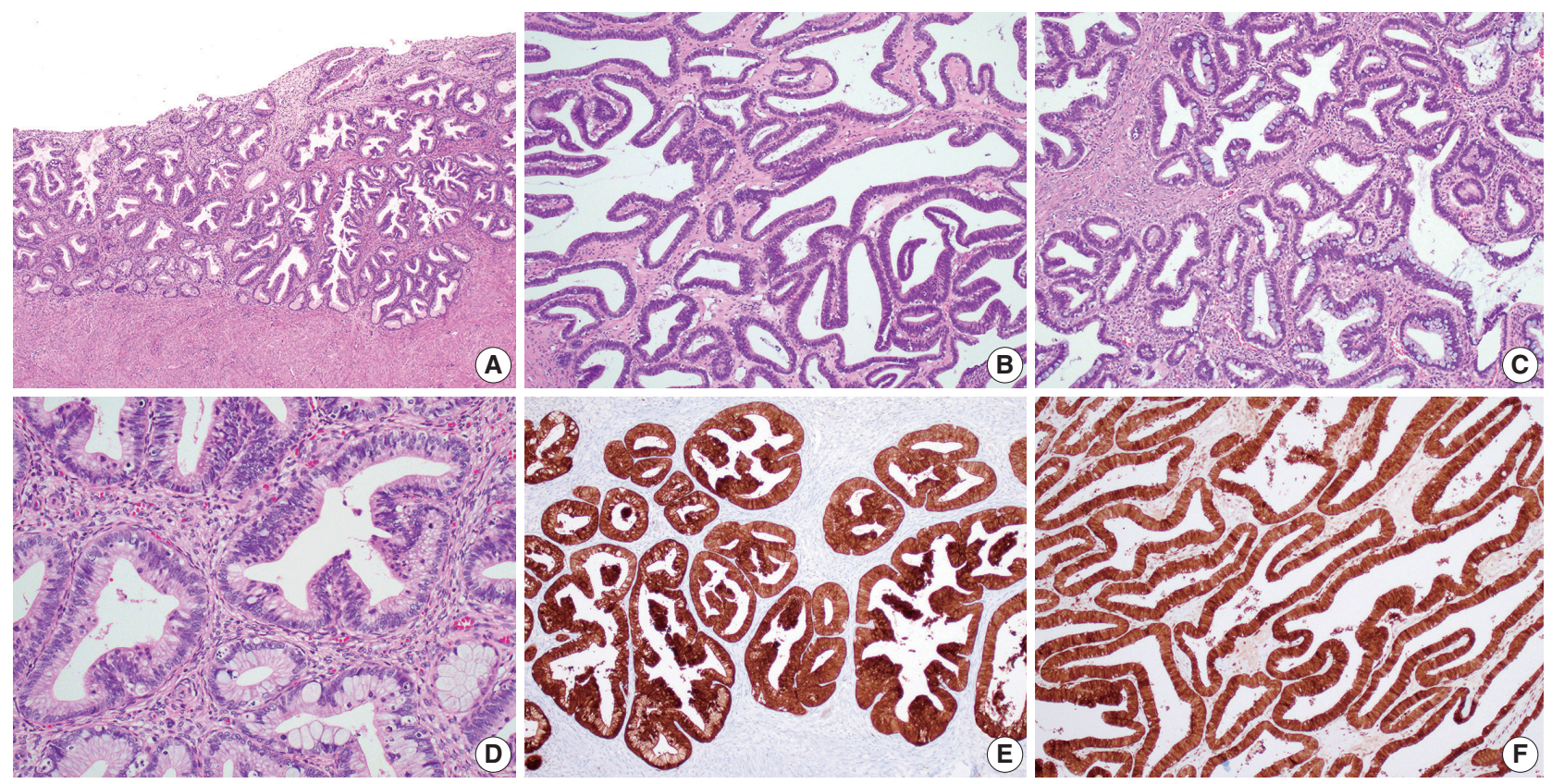

Fig. 1. (A) Histological findings of the endocervical lesion. Endocervical adenocarcinoma in situ (AIS) extensively involves the endocervix. No cervical stromal invasion is identified. (B-D) Histological findings of the ovarian lesion. The tumor displays a well-differentiated glandular architecture with primarily $(B)$ endometrioid-like and focally $(C)$ mucinous differentiation. (D) High-power magnification of the ovarian lesion reveals enlarged and hyperchromatic nuclei with intracytoplasmic mucin vacuoles, mitotic figures, and apoptotic bodies, similar to those of the endocervical AIS. (E, F) p16 immunostaining results. Both the (E) endocervical and (F) ovarian lesions show strong and diffuse p16 immunoreactivities.

cervical adenocarcinoma or in a primary lesion with extrauterine extension. ${ }^{6}$ However, there have been cases of early-stage disease, and even cases of noninvasive carcinoma, that involved the ovary. ${ }^{1,2,5,7}$ Most ovarian lesions show well-differentiated villoglandular and confluent glandular patterns, resembling those of primary ovarian borderline mucinous or endometrioid tumor. ${ }^{2}$ In a previous study, all cases examined were initially thought to represent independent primary ovarian epithelial tumors, including borderline tumors or grade 1 carcinomas of mucinous or endometrioid type. ${ }^{8}$ In cases with no stromal invasion in an endocervical adenocarcinoma, a borderline-appearing ovarian tumor could reasonably be interpreted as an independent primary ovarian tumor by the pathologist. ${ }^{7}$ In this situation, the presence of the identical HPV type in both the endocervical and ovarian tumors supports that the ovarian lesion is a metastatic endocervical adenocarcinoma rather than an independent primary ovarian tumor. Studies associating HPV and ovarian tumors have yielded almost universally negative results, suggesting that primary ovarian tumors are etiologically unrelated to HPV infection. ${ }^{7}$ In addition, p16 immunostaining may prove useful for distinguishing metastases from primary ovarian tumors. p16 immunoreactivity is a surrogate marker of high-risk HPV infection. ${ }^{9}$ Although p16 expression is absent or sporadically positive in ovarian mucinous and endometrioid carcinomas, HPV-related endocervical tumors typically display strong and diffuse nuclear p16 immunoreactivity. In conjunction with clinicopathological features, p16 expression in ovarian lesions is associated with high-risk HPV and supports the diagnosis of metastatic endocervical adenocarcinoma.

In summary, we present a case of endocervical AIS involving the ovary. Notably, minimal to no stromal invasion in endocervical adenocarcinoma does not exclude the possibility of metastasis to the ovary. Cases of concurrent endocervical AIS and ovarian tumor, although rare, prompt thorough sampling of the endocervix to exclude stromal invasion. Careful morphological evaluation and use of ancillary tests promote an accurate diagnosis. p16 immunostaining and HPV testing of ovarian lesions may be confirmatory if positive.

\section{ORCID}

Hyun-Soo Kim: http://orcid.org/0000-0002-2356-7822

Yeon Seung Chung: https://orcid.org/0000-0003-0197-2699

Moon Sik Kim: https://orcid.org/0000-0002-5804-8790

Hyang Joo Ryu: https://orcid.org/0000-0002-3629-929X

Ji Hee Lee: https://orcid.org/0000-0003-0054-7033 


\section{Author Contributions}

Conceptualization: HSK.

Data curation: YSC.

Formal analysis: YSC, MSK, HJR, JHL.

Funding acquisition: HSK.

Investigation: HSK, YSC.

Methodology: MSK, HJR, JHL.

Project administration: HSK.

Resources: HSK, YSC.

Software: HSK.

Supervision: HSK.

Validation: MSK, HJR, JHL.

Visualization: HSK.

Writing—original draft: HSK, YSC.

Writing—review \& editing: HSK, YSC, MSK, HJR, JHL.

\section{Conflicts of Interest}

The authors declare that they have no potential conflicts of interest.

\section{Acknowledgments}

This work was supported by the Korean Society of Pathology Grant (2016) and a faculty research grant of Yonsei University College of Medicine (6-2018-0049).

\section{REFERENCES}

1. Chang MC, Nevadunsky NS, Viswanathan AN, Crum CP, Feltmate $\mathrm{CM}$. Endocervical adenocarcinoma in situ with ovarian metastases: a unique variant with potential for long-term survival. Int J Gynecol Pathol 2010; 29: 88-92.

2. Turashvili G, Farmer P, Colgan T, Childs T. Human papillomavi- rus-related ovarian metastasis with endocervical adenocarcinoma: report of 2 cases and review of literature. J Low Genit Tract Dis 2015; 19: e60-3.

3. Yada-Hashimoto N, Yamamoto T, Kamiura S, et al. Metastatic ovarian tumors: a review of 64 cases. Gynecol Oncol 2003; 89: 314-7.

4. McCluggage WG, Wilkinson N. Metastatic neoplasms involving the ovary: a review with an emphasis on morphological and immunohistochemical features. Histopathology 2005; 47: 231-47.

5. Khor TS, Brennan BA, Leung YC, Sengupta S, Stewart CJ. Cervical adenocarcinoma metastatic to the ovary mimicking primary ovarian carcinoma. Pathology 2009; 41: 397-400.

6. Reyes C, Murali R, Park KJ. Secondary involvement of the adnexa and uterine corpus by carcinomas of the uterine cervix: a detailed morphologic description. Int J Gynecol Pathol 2015; 34: 551-63.

7. Ronnett BM, Yemelyanova AV, Vang R, et al. Endocervical adenocarcinomas with ovarian metastases: analysis of 29 cases with emphasis on minimally invasive cervical tumors and the ability of the metastases to simulate primary ovarian neoplasms. Am J Surg Pathol 2008; 32: 1835-53.

8. Elishaev E, Gilks CB, Miller D, Srodon M, Kurman RJ, Ronnett BM. Synchronous and metachronous endocervical and ovarian neoplasms: evidence supporting interpretation of the ovarian neoplasms as metastatic endocervical adenocarcinomas simulating primary ovarian surface epithelial neoplasms. Am J Surg Pathol 2005; 29: 281-94.

9. Vang R, Gown AM, Farinola M, et al. p16 expression in primary ovarian mucinous and endometrioid tumors and metastatic adenocarcinomas in the ovary: utility for identification of metastatic HPV-related endocervical adenocarcinomas. Am J Surg Pathol 2007; 31: 653-63 